\title{
Disfunciones informativas en la divulgación científica de las crisis ambientales acontecidas en España
}

\author{
Informational dysfunctions in the scientific disclosure of \\ environmental crisis occurrences in Spain
}

\author{
Aida María de Vicente Domínguez, Universidad de Málaga \\ Facultad de Ciencias de la Comunicación, Calle León Tolstoi, s/n, 29010 (Málaga) \\ aidamaria@uma.es | Orcid: http://orcid.org/0000-0002-8085-5097
}

DOI: http://dx.doi.org/10.12795/Ambitos.2019.i45.07

\begin{abstract}
Resumen
El objetivo de esta investigación es recopilar los déficits informativos que se dan en la cobertura de las crisis ambientales identificados en estudios empíricos precedentes. En concreto, se abordan los relativos a las fuentes, la edición y el enfoque temático de estos acontecimientos. Una pesquisa que ofrece un Statu Quo de estas malas praxis y cuyo conocimiento se considera de interés para usarlas como base metodológica en estudios comparativos futuros, impartirlas en materias relacionadas con el periodismo, la comunicación y la divulgación científica, e incrementar el conocimiento en esta línea de investigación. La metodología ha consistido en un acercamiento exploratorio a la producción académica a través de bases de datos nacionales e internacionales. Las conclusiones desvelan entre las principales deficiencias una elevada representatividad de fuentes oficiales, principalmente políticas, frente a una escasez de fuentes expertas
\end{abstract}


independientes, ausencia de pluralismo informativo, fuerte credibilidad hacia los centros de investigación del gobierno, errores gramaticales y de precisión en los conceptos medioambientales, enfoque temático centrado en el cruce de acusaciones entre las fuentes implicadas, elaboración superficial de la materia informativa prestando atención a los rasgos más atractivos o sensacionalistas y no a los riesgos para la salud humana y el medio ambiente.

\begin{abstract}
The object is to gather all of the informative deficiencies disclosed and alerted by previous scientific studies that are centered on the sources, the edition and the thematic focus of these doings. An inquest whose data is considered to be of interest to obtain a Status Quo of this malpractice broadens the knowledge of this material and can act as a methodological base for future comparative studies. The methodology consisted of an exploratory approach to Spanish academic production in the communication sector through a bibliographical search of national and international databases. The results indicate that among the principle deficiencies is an elevated representation of official sources, principally political sources, compared to a scarcity of independent expert sources and the absence of informative pluralism, strong credibility given to government run investigative centers, human sources dominate the documented ones, grammatical errors and errors in the precision of environmental concepts, thematic focus centered on the crossfire of accusations among the implicated sources, superficially elaborated without paying attention to the risk to human health or the environment but highlighting the most attractive and sensationalist features.
\end{abstract}

Palabras clave: divulgación científica, crisis ambientales, España, medios de comunicación, déficit.

Keywords: scientific disclosure, environmental crises, Spain, mass media, deficit.

\title{
1. INTRODUCCIÓN
}

Las crisis ambientales son los acontecimientos más representativos en el "ámbito de las coberturas y representaciones del medio ambiente" (Barranquero y Marín- García, 2014 , p. 484). $Y$ es que se trata de acontecimientos que tienen un perfil de riesgo, incertidumbre y preocupación, por sus consecuencias inmediatas, próximas y futuras para el ecosistema. Acontecimientos que están teñidos de peligrosidad y vulnerabilidad $^{1}$. Sin embargo, la construcción de su discurso informativo presenta diversas disfunciones que han sido desveladas y corroboradas por diversos investigadores. Tómese como referencia las aportaciones de Montaño (1999); 
Fernández-Reyes (2001); Elías (2001, 2003 y 2006); Humanes y Cheng (2002); González-Villariny (2008); Vicente (2010); Odriozola (2010 y 2011).

Unas disfunciones que pretenden aunarse en este estudio con el objeto de reflexionar sobre las medidas y enfoques que deben reforzarse desde el ámbito académico para paliarlas y formar a comunicadores con capacidad para convertirse en gestores del derecho de los ciudadanos para que estén bien informados y fomenten su capacidad crítica.

La identificación de las inexactitudes como método de análisis para alcanzar el rigor en la información o conocer la verdad de un acontecimiento fue el seleccionado por el profesor Verón (1983), hace ahora casi 40 años, para analizar cómo los medios de comunicación franceses abordaron el acontecimiento del accidente producido en 1979 en la central nuclear estadounidense de Three Mile Island. Mediante una radiografía de lo acontecido y el análisis del discurso de la información de los medios consultados, Verón recompuso el acontecimiento y explicó la verdadera importancia y gravedad de los hechos por encima de las disfunciones cometidas.

En esta investigación se pretende abordar los déficits en la divulgación científica de las crisis ambientales desde tres enfoques: las fuentes, la edición y el enfoque temático. Una recopilación no abordada hasta el momento y que pretende captar más afinadamente esta realidad que ha sido observada y analizada, en ocasiones atendiendo a unas mismas variables de estudio y en otras divergentes, con el objetivo de agrupar todas estas carencias. Un estudio que también se considera significativo porque las carencias informativas resultantes servirán como material académico, para intentar paliarlas, y como base metodológica en pesquisas futuras para comprobar si continúan practicándose las malas praxis alertadas por los investigadores.

El periodista de la BBC en Londres, Martín Redfern declaró: "los periodistas son lo único que tiene el público para poder comprender un aspecto de las cosas y esto nos da una importancia y una responsabilidad significativas", (como se cita en Calvo, 1990, p.15). Una responsabilidad que en el caso de las crisis ambientales debe incrementarse para ofrecer una divulgación científica alejada de la manipulación, la sobreinformación o la torpeza informativa.

\section{OBJETIVOS Y METODOLOGÍA}

El objetivo general de la investigación es identificar cuáles son las disfunciones en la divulgación científica de las crisis ambientales en los medios de comunicación generalistas españoles. Por ello, el campo de estudio se ha centrado en la cobertura de las dos principales crisis ecológicas acontecidas en España: el desastre de Aznalcollar (1998) y del Prestige (2002). En concreto la investigación se centra en: 
- Investigar los déficits en las fuentes del discurso informativo.

- Identificar las disfunciones en la redacción y edición de las piezas informativas.

- Indicar las inexactitudes en el encuadre o enfoque temático en estas coberturas.

Un estudio cuya metodología se estructura en los siguientes pasos:

- Revisión bibliográfica en bases de datos nacionales e internacionales (Dialnet, Rebiun, Teseo, TDR, B-on y Google Academic). Las ecuaciones de búsqueda usadas han sido siete: "comunicación ambiental", "periodismo ambiental", "crisis ambiental", "catástrofe ambiental", "información ambiental" "Doñana" y "Prestige". Ecuaciones de búsqueda que se han ido combinando.

- Los estudios científicos recopilados se han regido por un criterio de selección: que sean investigaciones que analicen cómo los medios de comunicación generalistas españoles dieron cobertura a estos acontecimientos y que aporten deficiencias vinculadas con las fuentes, la edición y el enfoque temático. Ergo, aquellos estudios que han analizado estas crisis ambientales y que no han estudiado o identificado déficits informativos relacionados con las variables objeto de estudio en un medio de comunicación generalista no forman parte del cómputo.

- Organización sistemática de la documentación seleccionada indicando de cada investigación: año, autor, título, tipo de artículo, y déficits informativos desvelados. En concreto, el corpus está conformado por los estudios de los siguientes investigadores: Montaño (1999), Fernández-Reyes (2001); Elías (2001, 2006 y 2008); Humanes y Cheng (2002); Agraso, Eixeras y Jiménez-Aleixandre (2003); González-Villariny (2008); Vicente (2010); Odriozola (2010 y 2011).

- Análisis total de las unidades de registro, no optando por una muestra aleatoria simple, con el objeto de obtener una panorámica del tema abordado lo más amplia posible. $Y$ es que el descarte de un estudio científico puede implicar la omisión de un déficit en la cobertura de las crisis ambientales al no haber sido analizado por otros investigadores.

- Unificados por categorías se procede a realizar el nivel de proximidad entre los diversos déficits informativos obtenidos y la identificación de cada uno de ellos.

- Discusión de los resultados y conclusiones. 


\section{RESULTADOS}

La unificación de las disfunciones identificadas en estudios precedentes está conformada por más de una decena de carencias informativas (relacionadas con las fuentes, la edición y el enfoque temático) que obstaculizan una divulgación científica correcta de las crisis ambientales.

\subsection{Disfunciones en las fuentes del discurso informativo}

Los resultados desvelan un uso muy elevado de fuentes institucionales para informar de las crisis ambientales. Así lo han confirmado diversos estudios (Humanes y Cheng, 2002; Agraso, et al., 2003; Elías, 2008; Odriozola, 2010; Vicente, 2010). Estudios que en su conjunto muestran como esta disfunción es practicada tanto por la prensa nacional como regional. Una elevada representatividad que no sólo se da en el cuerpo de la noticia sino también en los titulares (Fernández-Reyes, 2001) y que suele estar conformada por las declaraciones de las administraciones vinculadas a la catástrofe cuya práctica totalidad corresponde a los cargos políticos (Odriozola, 2010). Un discurso que suele aumentar al inicio de las informaciones lo que las "caracteriza como generadoras de textos periodísticos" (Odriozola, 2011, p. 90).

Una disfunción que no permite conocer la verdad del acontecimiento porque las declaraciones de las fuentes implicadas están asesoradas por sus gabinetes de comunicación para ofrecer un discurso que les sea favorable. En este contexto, significativas son las aportaciones de Corbacho y Ruas (s.f.) al desvelar que en la crisis del Prestige se aportaron "una serie de desafortunadas declaraciones que sólo sugerían intentos de ocultación, manipulación y desinformación, entendida esta última como un deliberado intento por difundir informaciones falsas, a sabiendas de lo que eran" (p. 4). Una manipulación del discurso que también, describe Esteban (1998), ocurrió en las declaraciones aportadas por estas fuentes en la crisis de Doñana al ofrecer "la más triste y contundente de las demostraciones imaginables acerca de la irresponsabilidad de gestión y la deliberada política de ocultamiento y falseamiento de la realidad" ( $p$. 1), no solo ocultando los datos sino "neutralizando o bloqueando las denuncias reiteradamente presentadas por las organizaciones ecologistas" (p. 1).

- La escasez de fuentes expertas fiables es otra de las disfunciones identificadas por estudios precedentes. Ergo, "aunque existe la creencia generalizada de que [...] pueden aportar conocimientos objetivos y juicios independientes" (Fernández-Parrat, 2006, p. 113) su representatividad es escasa como fuente de información. Una escasa representatividad que tan sólo suele estar presente de forma puntual al inicio de la crisis porque se recurre a estas de manera continuada, si bien, "no existe un contacto que se mantenga a lo largo del tiempo" (Odriozola, 2010, p. 345). Y significativa es la 
disfunción desvelada por Elias (2008). Se trata del uso de fuentes expertas que denomino encubiertas. En concreto, identifica dos modalidades:

-El uso de fuentes expertas que trabajan en centros que dependen de las instituciones implicadas cuyas declaraciones están controladas por el gobierno. Unas fuentes a las que recurren los profesionales de la información porque "piensan que lo que declara un científico o un ingeniero o cualquier otro tipo de técnico de la administración es una verdad científica o técnica" (pp. 38-39). De hecho, ni en la crisis de Doñana ni del Prestige "se cuestionó en los medios de comunicación el modelo científico en el que el CSIC tiene una alta vinculación política" (Elías, 2006, p. 189). Es más, en el caso de Doñana, el índice de credibilidad de esta institución "Ilegó hasta tal punto que los procesos de retirada de lodos que no se habían realizado siguiendo los consejos del CSIC- fueron- denunciados por los medios de comunicación" (Elías, 2001, p. 298).

-El uso de fuentes expertas nombradas por el gobierno para gestionar la comunicación con los medios de comunicación. $Y$ es que los gobiernos suelen crear un comité de expertos para que emitan comunicados que alimenten a periodistas generalistas que confían en la aparente credibilidad de estos especialistas nombrados a dedo por el gobierno para que ofrezcan una información favorable y supervisada por estos. Un comité compuesto incluso por expertos no especializados o cualificados. Una acción denominada estrategia de comunicación política a través de fuentes expertas (Elias, 2008) o strategy of instrumentalising scientific expertise (Elías, 2007) que también se encarga de atacar a otros científicos que pretendan desacreditarle.

Unos expertos que se brindan, desvela Elías (2007), porque es una estrategia de patrocinio político de las catástrofes: a cambio de una relación leal se les recompensa con el puesto de dirección en importantes instituciones científicas como el CSIC, la NASA en los Estados Unidos o la Agencia Espacial Europea. Unas posiciones que no son seleccionadas por los científicos sino por los gobiernos. Ergo, se les ofrece una carrera científica.

Montero (1998) ya advertía que si "la fuente ideal dentro de la administración son sus técnicos [...] estos suelen estar sometidos a la férrea disciplina comunicativa del organismo en cuestión" (p. 4). Por ello, deben prevalecer las voces científicas independientes procedentes de las universidades al ser las más cualificadas, aunque en la práctica como desvelan Agraso et al., (2003) son las más escasas. Una falta de fuentes independientes, tanto por la administración como por los medios, que fue puesta de manifiesto por los científicos independientes publicando una carta en la revista Sciencie.

-La escasa presencia de grupos contrarios a las fuentes oficiales o de otros actores perjudicados por la crisis es otro de los déficits identificados. Agraso et al., (2003) 
destacan la escasa presencia de "los sectores directamente afectados por el vertido" (p. 5) y Vicente (2010) concluía que priman los aspectos positivos "silenciando a grupos de ciudadanos contrarios a la gestión política y complementando una visión muy cercana a los intereses gubernamentales" (p. 251). Una carencia también detectada en estudios generales sobre la información medioambiental cuyos resultados desvelaron: "la sociedad civil y las ONG, como agentes, movilizadores, así como los expertos, no equilibran la presión de los intereses económicos o políticos en torno al medio ambiente" (Rodríguez y Bezunartea, 2015, p. 99).

Esteban (1998) incluso considera que si en el caso de Doñana las organizaciones ecologistas hubiesen tenido mayor representatividad en los medios "pronto hubiera sido imposible ocultar el verdadero comportamiento de cada institución" (p.3). Y muestra un ejemplo de su escaso interés mediático:

Basta señalar que la manifestación de varios miles de personas celebrada en Sanlúcar de Barrameda el Domingo 10 de mayo, que había sido convocada por todo el movimiento ecologista estatal y había recibido innumerables apoyos del movimiento ecologista internacional (miles de cartas habían sido ya enviadas desde todo el mundo por correo y por Internet al Presidente de la Junta de Andalucía y a otras autoridades), no apareció en los telediarios de ninguna de las cadenas de televisión estatales ni fue reseñada en ninguno de los grandes telediarios de difusión estatal (como excepción, un diario catalán le dedicó dos líneas). Pocos días después, una manifestación mucho menos numerosa de los mineros de Aznalcollar pidiendo la reapertura inmediata de la mina fue recogida en todos los medios, sin excepción, incluyendo en muchos diarios una fotografía del evento (p.4).

\subsection{Disfunciones en la edición}

El principal déficit detectado en el proceso de redacción y edición de estas piezas informativas es la falta de precisión en los conceptos. Fernández-Reyes (2001) detectó en su estudio sobre la cobertura de Doñana un recorte de los nombres completos. Un ejemplo fue "denominar al Parque Natural de Cazorla, Segura y Las Villas como Parque Natural de Cazorla" (p. 192). Y "entre las confusiones más generalizadas estuvo la de no diferenciar el Parque Natural Entorno de Doñana, que fue atravesado por la ola contaminante, del Parque Nacional de Doñana que fue protegido con muros de contención" (p. 332), o utilizar el término Parque de manera sesgada para referirse a "el Parque Nacional" (p. 332). Errores no sólo de imprecisión de los conceptos sino también sintácticos en el uso de las mayúsculas y minúsculas. 
Montero (1994) ya alertaba de estas imprecisiones que décadas después aún siguen produciéndose: "¿Cuántas veces se confunden en los medios de comunicación «Parques Nacionales» con «Parques Naturales»? ¿Cuántos periodistas consideran que el agujero de ozono y el efecto invernadero son la misma cosa?" (p. 95).

Una disfunción que también fue puesta de manifiesto en la cobertura del Prestige. En concreto, Elías (2006) aporta otro déficit en el uso de la terminología que consiste en acatar y usar el concepto utilizado por las autoridades. Autoridades que lo usan con el objeto de minimizar la dimensión de la catástrofe:

Fue curioso observar cómo en los primeros días las autoridades, tanto científicas como políticas, insistían a los periodistas en que usaran el término fuel, en lugar de petróleo o crudo [...] Pero lo que llevaba el Prestige era "fuel del tipo número 6 ", en la terminología inglesa. Es decir, el peor de todos porque es viscoso -con lo que cuesta eliminarlo- $y$, además, tenía sustancias potencialmente cancerígenas (p. 172).

Una estrategia para omitir la verdadera gravedad de los hechos que también fue usada, como desvelan Corbacho y Rúas (s.f) para evitar en los primeros días del desastre del Prestige que se usara el concepto "marea negra", cuando la propia WWF/ADEMA calificó "la catástrofe como la peor marea negra de la historia, tras la tristemente protagonizada por el Exxon Valdez en Alaska hace más de una década" (p. 1).

\subsection{Disfunciones en el encuadre de la pieza informativa}

Los resultados desvelan que es limitado el espacio dedicado a las consecuencias relevantes como las que afectan al medio ambiente (Vicente, 2010). Si bien, el discurso que domina en la cobertura de las crisis ambientales es el político, como concluyen González-Villariny (2008) y Odriozola (2010) independientemente del enfoque temático que se aborde (González-Villariny ${ }_{2} 2008$ ).

Una visión política que no tendría "porqué desvirtuar la estrategia informativa de un medio; ya que, dentro de esas consecuencias, se encuentran aspectos de gran interés como las reformas en política medioambiental, reformas en la política marítima o la creación de comisiones de investigación" (Odriozola, 2010, p. 340). Sin embargo, no son estas las repercusiones que se abordan porque se centran en el cruce de acusaciones entre las administraciones públicas en la catástrofe ambiental: lo desveló Montaño (1999) y el estudio de Odriozola (2010) lo corroboró una década después.

Una disfunción que además suele enfocarse en la "confrontación de ideas y valores e intereses en juego" (Lorente, 2014, p. 184) que muestra la falta de consenso entre políticos. Encuadre político que cuando aborda los debates políticos, concluye 
González-Villariny (2008), se centra en "los errores y las negligencias cometidas por el gobierno y otras polémicas surgidas" (p. 217) siendo el resto de las actividades a las que presta más atención la gestión política y las protestas multitudinarias en la categoría política. En este contexto, significativas son las críticas de Corbacho y Ruas (s.f) sobre la postura de las fuentes institucionales en el Prestige:

Que un problema de gobierno se convirtió en un problema de partido frente a la oposición, es decir, que el gobierno dio la sensación de estar más interesado en reaccionar principalmente en clave partidista, lo que derivó tristemente en una lucha encarnecida y un intercambio de declaraciones, en lugar de unir los esfuerzos para paliar una catástrofe sin precedentes en Galicia (p. 4).

Una cobertura que, como indican los estudios, además de no centrarse en los riesgos para la salud humana o medio ambiente, otorga "prioridad a los aspectos más sensacionalistas y atractivos del suceso" (González-Villariny, 2008, p. 217). Prácticas que provocan un desequilibrio informativo, una información descontextualizada y de poca calidad. Datos sobre el que ya alertó Montaño (1999) y que coinciden con los obtenidos en otros estudios generales sobre la cobertura de las crisis ambientales. Lorente (2014) desvela que son informaciones sin tiempo para el análisis cuya superficialidad está lejos de animar a la participación ciudadana, y Montero (1994) advertía que no se abordan las conexiones entre los problemas ambientales y sus efectos en el medio humano, sino que se centran en "conflictos más o menos coyunturales y a veces hasta anecdóticos” (p. 93). En este contexto, significativa es la exposición de Lozano (2016):

Así pues, más de un siglo más tarde de aquel famoso hundimiento del Titanic, las noticias de hoy en día sobre riesgos y catástrofes se caracterizan por seguir siendo narraciones sensacionalistas, más bien espectaculares, llamativas, mal estructuradas y, en muchas ocasiones, superficiales. No informan con un nivel deseable de rigor (pertinencia, contrastación y completitud), ni suelen ofrecer datos totalmente ciertos (descripciones y argumentos), más bien conducen a la ambigüedad y al desconcierto de los lectores y, por consiguiente, el púbico no consigue aprender por esta vía las nociones básicas sobre los riesgos y catástrofes que les permitieran identificar (saber) y prevenir (saber hacer en) los futuros acontecimientos catastróficos de similares características. (Cfr. Lozano Ascencio, C., 2015), (p. 47).

- Otra disfunción identificada por Vicente (2010) se centra en las elevadas intervenciones en directo de los noticieros "que no proporcionan más valor a la crónica que el hecho de que la audiencia pueda comprobar que el equipo de periodistas realmente se encuentra presente en el lugar de los hechos" (p. 255) por lo que indica la necesidad de un replanteamiento de las conexiones en directo porque "la precipitación que 
acompaña a la información de última hora suele ser una fuente de imprecisiones que podría ahorrarse" (p. 255). Una necesidad formativa también compartida por otros investigadores:

A todo esto hay que añadir otra dificultad, y es cuando el medio en cuestión es una televisión. La falta de imágenes que pueden ayudar a explicitar un tema en concreto supone una inversión de doble esfuerzo para el periodista, porque no debe limitarse a contar lo que sabe, sino que, además, antes de confeccionar su noticia o reportaje debe plantearse una o varias situaciones audiovisuales que servirán para explicar el hecho en cuestión. (Soriano, 2002, p. 129)

\section{CONCLUSIONES}

El objetivo de la investigación ha sido recopilar los déficits informativos en la cobertura de las crisis ambientales en España identificados en estudios precedentes y que obstaculizan una correcta divulgación científica. En concreto, se ha indagando en las deficiencias producidas con las fuentes, el enfoque informativo y la edición o redacción.

Las conclusiones obtenidas ponen de relieve que se siguen produciendo los defectos de calidad y cantidad que como ya alertaba Montero (1994), eran objeto de queja por parte de quienes se dedican a las tareas de divulgación o conservación de la naturaleza. Y que criticaban tanto la falta de objetividad como de información que ayudasen a conocer la interconexión entre "las diferentes decisiones/actuaciones/fenómenos relacionados con el medio natural [...] y las repercusiones de todo orden (social, económico, medioambiental...) que la decisión/actuación/fenómeno pueda conllevar" ( p. 92). Y las disfunciones por las que se producen estas carencias en el tratamiento de estos hechos son las siguientes:

- Elevada presencia de fuentes instituciones, principalmente de los cargos políticos implicados, tanto en los titulares como en el cuerpo de la pieza informativa. Lo que implica que la información se agota en la noticia puntual, en un periodismo de declaraciones oficialistas, que no contribuye a su profundización desde una perspectiva científica. Ergo, es necesario contrastar adecuadamente estas informaciones para que los mass media no terminen convirtiéndose en "sus portavoces, inconsciente o conscientemente" (Rekondo, 2002, p. 421).

- Escasez de fuentes expertas independientes universitarias frente a la presencia de fuentes expertas encubiertas porque trabajan en centros de investigación controlados por el gobierno o aceptan formar parte del comité de expertos para crear informes y dar declaraciones favorables a las fuentes implicadas. Lo que impide una correcta divulgación científica e implica la necesidad de formar a los profesionales de la información para que identifiquen si una fuente experta tiene vinculación con la fuente impli- 
cada y para que conozcan herramientas que les permitan acceder a fuentes fiables como: las guías de expertos de las universidades, The Global Expert, la guía de expertos Fundación Descubre, o los encuentros entre expertos y periodistas organizados desde diversas asociaciones de periodismo ambiental. $Y$ es que las fuentes fiables tienen escasa visibilidad: su presencia se usa al inicio del acontecimiento pero no es continua en el tiempo.

- Las voces críticas no tienen el mismo espacio o tiempo de intervención, lo que genera un potente mecanismo de desinformación. Una falta de pluralismo que priva a los ciudadanos de la verdad. $Y$ es que a través de las fuentes con las se construye el discurso informativo el colectivo social se posiciona, aumenta o disminuye su grado de implicación o concienciación, así como su grado de entendimiento sobre las causas que originan las crisis medioambientales, lo que permite crear juicios de forma crítica y actitudes responsables comprometidas.

- Falta de precisión en los conceptos clave de una crisis ambiental confundiendo unas terminologías con otras, utilizando los conceptos de manera sesgada, recortando los nombres, mezclando las mayúsculas con las minúsculas. Disfunciones que implican la necesidad de adoptar medidas formativas para ser rigurosos con los datos, sobre todo, con los conceptos principales de una pieza informativa. Por ello, es necesario aportar recursos de utilidad como los diccionarios especializados y concienciar de la importancia de comprobar los conceptos. Una imprecisión que implica no sólo una divulgación errónea, sino que los ciudadanos terminen adoptando los conceptos que se plasman en los medios de comunicación.

- Acatar y usar la terminología aportada por las fuentes implicadas en la catástrofe sin contrastarla. Un uso que también implica un blanqueo activo de las consecuencias de la catástrofe usando terminologías que enmascaren las verdaderas consecuencias, al tiempo, que consiste en ofrecer una divulgación errónea de la verdadera gravedad de los hechos.

- Los ángulos temáticos se centran principalmente en el político, en el cruce de acusaciones entre las fuentes implicadas sobre los errores o las negligencias, y en los aspectos más sensacionalistas de las crisis ambientales. Ergo, el espacio al medio ambiente queda limitado. Lo que implica que no hay interés por la divulgación científica. Los ciudadanos no pueden entender el por qué, las causas, las consecuencias y las posibles soluciones para estos acontecimientos.

- En estudios futuros se realizarán grupos de discusión con periodistas generalistas y especializados, que aborden la información ambiental, para conocer las causas que expliquen estas deficiencias y abordar posibles soluciones mediante el diseño de propuestas didácticas. 
- Estudios futuros podrán usar estas deficiencias como base metodológica para analizar si continúan produciéndose estos déficits en los medios generalistas españoles; para estudiar si están presentes en la cobertura de las crisis ambientales en otras áreas geográficas; y para comprobar si se dan en los medios de comunicación especializados en medio ambiente o si su procedencia es exclusiva de los generalistas.

\section{Notas}

${ }^{1}$ El término peligrosidad se refiere al fenómeno o actividad que puede producir pérdida de vidas humanas, heridos $u$ otros impactos en la salud como daños y pérdida de bienes, infraestructuras, servicios o daños ambientales. La peligrosidad depende de factores meteorológicos, geológicos o hidrológicos. Por otro lado, el concepto de vulnerabilidad se refiere a las características y circunstancias de una comunidad, sistema o bien que lo hace susceptible a los efectos dañinos de la amenaza. El concepto de vulnerabilidad es muy amplio y abarca desde el uso que se haga del territorio hasta la estructura de los edificios y construcciones, y depende fuertemente de la respuesta de la población frente al riesgo (Brilly y Polic, 2005). (como se cita en Llasat, Llasat y Cortés, 2016, pp. 787-788).

\section{Referencias}

Agraso, M.F. \& Eirexas, F. \& Jiménez- Aleixandre, M. P. (2003). El prestige en la prensa gallega en el 2002: um análisis preliminar. Quark: Ciencia, medicina, comunicación y cultura, 28-29, 1-10.

Barranquero, A. \& Marín-García, B. (2014). La investigación en comunicación y periodismo ambiental en España. Prisma social, 12, 474- 505.

Brilly, M. \& Polic, M. (2005). Public perception of flood risks, flood forecasting and mitigation. Natural Hazards and Earth System Sciences, 5, 345-355. doi: https://doi.org/10.5194/nhess-5-345-2005

Calvo, M. (1990). Ciencia y Periodismo. Barcelona: Centro de Estudios para el Fomento de la Investigación.

Corbacho, J.M y Ruas, J. (Sin fecha). La comunicación de crisis: el caso del Prestige. Recuperado de https://bit.ly/2XDfdta

De Pablos, J.M. \& Elias, C. (2003). CSIC: chapapote de la ciencia española o como se manipula la información en catástrofes ecológicas. Revista Latina de Comunicación social, 53. Recuperado de https://bit.ly/2PuTzEr

Elías, C. (2007). The use of scientific expertise for political PR. The "Doñana" and "Prestige". Cases in Spain. In M.W. Bauver y M. Bucchi (ed.), Journalism, Science and Society. Science Communication between news and Public relations (pp. 227-238). New York/ London: Routledge. 
Elías, C. (2001). Periodismo especializado en medio ambiente: el caso Doñana como paradigma de manipulación informativa. Ámbitos, revista Internacional de Comunicación, 6, 297-303.

Elías, C. (2006). Medio ambiente, manipulación política y control del riesgo: análisis del caso del hundimiento del petrolero Prestige. Ámbitos: revista Internacional de Comunicación, 15, $171-190$.

Elías, C. (2008). Fundamentos de periodismo científico y divulgación científica. Madrid: Alianza editorial.

Esteban, J. A. (1998). Los lodos tóxicos de Aznalcóllar y el desarrollo sostenible de Doñana. Recuperado de https://bit.ly/2UyXXDn

Fernández-Parrat, S. (2006). La información ambiental en los medios de comunicación. Dificultades y retos. Telos, 68,110-115.

Fernández-Reyes, R. (2001). Periodismo ambiental y ecologismo: tratamiento informativo del vertido de Aznalcollar en El País, edición de Andalucía (1998- 1999) (Tesis doctoral). Facultad de Ciencias de la Información de la Universidad de Sevilla, Sevilla.

González-Villariny, N. (2008). La comunicación de riesgo en la prensa escrita: un estudio del tratamiento informativo del naufragio del petrolero Prestige en los diarios El País y el Mundo (Tesis doctoral). Facultad de Ciencias de Información de la Universidad Complutense de Madrid, Madrid.

Humanes; M.L. \& Cheng, L. (2002). La información sobre riesgos medioambientales. El caso de la contaminación del parque de Doñana. En B. Gutierrez (Ed.). Medios de Comunicación y medio ambiente (pp. 97-107). Salamanca: Universidad de Salamanca.

Llasat, M. \& Llasat, M.C. \& Cortes, M. (marzo de 2016). De la prensa a las apps. Un recorrido por la comunicación de los riesgos naturales en la prensa escrita y el papel de las nuevas tecnologías. I Congreso Internacional Comunicación y Pensamiento. Comunicracia y desarrollo social, Departamento de Periodismo II de la Universidad de Sevilla: Sevilla.

Lorente, J. I. (2014). La gestión informativa del riesgo ambiental en tiempo de crisis. Cuadernos de información y comunicación, (19), 179- 194.

Lozano, C. (septiembre de 2015). La construcción del mensaje sobre los riesgos y catástrofes de origen natural en los noticiarios de las principales cadenas de televisión de cobertura nacional en España (2013 - 2014). Seminario Internacional sobre Ciencias Sociales y Riesgo de Desastre en América Latina: un encuentro inconcluso. Université de Buenos Aires, Argentina.

Lozano, C. (2016). El sensacionalismo de los riesgos y las catástrofes: un siglo en los relatos periodísticos. Revista Internacional de Historia de la Comunicación, 5 (1), 35-51. doi: http://dx.doi.org/10.12795/RiHC.2015.i05.03

Montaño, M. (1999). La catástrofe ecológica de Aznalcóllar de 1998: un reto para el periodismo especializado en información general. En Reig, R. y Ruiz, M. J. (coords.), Medio de comunicación y acontecimientos del siglo XX (pp. 149- 159), Sevilla: Universidad de Sevilla. 
Montero, J. M. (1994). Medios de comunicación y medio ambiente. En R. Mendoza (coord.), Actas de la VIII Aula de Ecología Educación Ambiental (pp. 89-101). Almería: Instituto de Estudios Almerienses.

Montero, J. M. (1998). Las fuentes de la noticia ambiental. Recuperado de https://bit.ly/2UuzLID

Odriozola, J. (2010). La crisis del Prestige en la prensa vasca desde una concepción ambiental. Estudios sobre el mensaje periodístico, 16, 331-347.

Odriozola, J. (2011). La construcción informativa de la catástrofe del Prestige: el abuso de las fuentes institucionales. Análisi, 44, 77-92.

Rodríguez Cruz, I. \& Bezunartea, O. (2015). El medio ambiente, supeditado a la conveniencia de la economía y la política. Diagnóstico de la información a partir de sus fuentes. Zer: Revista de estudios de comunicación, 39 (20), 85-100. doi: 10.1387/zer.15523.

Soriano, G. (2002). El tratamiento de los problemas ambientales en una televisión estatal. En. B. Gutiérrez (Ed.), Medios de comunicación y medio ambiente (pp. 127- 134). Salamanca: Universidad de Salamanca.

Vicente, M. (2010). Agendas, encuadres y discursos en los noticieros televisivos españoles durante la crisis del Prestige. Athenea Digital: revista de pensamiento e investigación social, 19, 249-257.

Verón, E. (1983). Construir el acontecimiento. Barcelona: Gedisa. 